First publ. in: Transport Theory and Statistical Physics 24 (1995), 6, pp. 855-880

\title{
MCT RESULTS FOR A SIMPLE LIQUID AT THE GLASS TRANSITION
}

\author{
M. Fuchs \\ Physik-Department \\ Technische Universität München \\ 85747 Garching, Germany
}

\begin{abstract}
The results of the mode coupling theory (MCT) for the slow relaxation processes in a liquid of hard spheres close to the liquid to glass transition are discussed. It is also shown that the MCT makes quantitative statements about the glassy structure, the relaxation processes in an intermediate time window and the final relaxation in the liquid phase. These results demonstrate the physical mechanism described by the idealized MCT and can be used to test the approximations of the theory experimentally.
\end{abstract}

\section{Introduction}

The dynamics of dense liquids exhibits slow processes which one would like to understand from the underlying microscopic parameters of the system. As the equilibrium structure of simple liquids is relatively well understood [1] a connection of the dynamics to the static structure appears desirable. The local structure of liquids is dominated by the excluded volume effect. Due to the hard core repulsion each particle possesses a volume which cannot be entered by other particles. A liquid of hard spheres with diameter $d$ is a simple theoretical model exhibiting this effect. The density $n=N / V$ of this liquid can be adjusted by constraining $N$ spheres to a volume $V$. The properties of this system in thermodynamic equilibrium handily depend on one parameter only, the packing fraction $\varphi=\frac{\pi}{6} n d^{3} . \varphi$ measures the 
relative volume belonging to one sphere and can be varied in between $\varphi=0$ and $\varphi \approx 0.64$ the value for random close packing if crystallization is prevented [2]. The equilibrium structure is well understood whereas the long time dynamics of a dense liquid of hard spheres has been studied theoretically only recently. Some of the new results shall be discussed below. The theoretical frame for these studies is provided by the mode coupling theory (MCT) of the liquid to glass transition whose general contents is covered in more detail in the reviews $[3,4,5]$. This article discusses various results of the $\mathrm{MCT}$ focussing on the quantitative, predictive nature of the MCT for simple liquids. Not the more universal results like the existence of power law relaxations and scaling laws shall be discussed in this article but the specific results which demonstrate the underlying physical mechanism in a simple liquid.

Only the so called idealized MC'T will be discussed which deals with the cage effect also known from the dynamics of liquids of intermediate density $[1,6]$. A particle is trapped in the cage formed by its neighbors. Each one of the neighboring particles again is trapped by its neighbors and a movement of one of the particles is possible only if the cages rearrange cooperatively. This feedback mechanism slows down the dynamics drastically if the particle density is increased.

In equilibrium, the local structure, i.e. the existence of a well defined shell of next neighbors, is dominated by the hard core repulsion. This shows up in the density fluctuations $\varrho_{\vec{q}}=\int d^{3} r e^{i \vec{q} \vec{r}}(\varrho(\vec{r})-n)$ as measured by the static structure factor $S_{q}=\frac{1}{N}\left\langle\varrho_{-\vec{q}} \varrho_{\vec{q}}\right\rangle$ on the length scale of the inverse average particle distance $q \approx 2 \pi / d$. For rather general interaction potentials the shape of $S_{q}$ around its peak at $q_{0}$ can be parametrized within an effective hard sphere liquid [1]. Therefore in a liquid of hard spheres, one can expect to study the essence of the cage effect, which as well is dominated by correlations on the average inter-particle distance [4].

Generally, it can be assumed that the so called hopping processes which open another decay channel for density fluctuations are present in a dense liquid of hard spheres as well. Within the extended MCT the range of applicability of the idealized MCT can be determined. One finds that the results of the idealized MCT remain valid for densities $\varphi$ below a transition region around a critical packing fraction $\varphi_{c}$. For densities close and above $\varphi_{c}$ hopping processes lead to qualitative changes which, in an intermediate time window, have been worked out [7]. The results of the idealized MCT can, however, not only serve as a first approximation to the more complicated extended theory. Moreover, there appear to be experimental systems where hopping corrections can be neglected. Colloidal suspensions of hard spheres 
have been studied by dynamic light scattering and within experimental uncertainty no indications of the corrections due to the hopping processes have been found [8].

In summary, the hard sphere liquid is ideally suited in order to study the consequences of the cage effect as described by the idealized MCT. Its equilibrium structure is well understood and depends on one external parameter $\varphi$ only. The Verlet-Weis approximation for the static structure factor $S_{q}(\varphi)$ has been checked by molecular dynamics results up to densities of $\varphi \approx 0.5$ [1]. Starting with the work of Boltzmann and Enskog the dynamics of a dilute system of hard spheres has been treated extensively $[1,6]$. An experimental system has been found whose equilibrium properties agree with that of the theoretical model [2]. Interestingly, this system consists of mesoscopic hard spheres dispersed in a solvent and is studied by static and dynamic light scattering; see the contribution of van Megen and Underwood in this volume.

This article discusses the existence of glass transition singularities in the idealized MCT in section 2 . In section 3 , the dynamics in an intermediate time window, the so called $\beta$-process, is characterized. In this dynamical window, a separation of spatial and temporal dependences is obtained as an universal result of the MCT. The spatial variation will identify the physical mechanism leading to the $\beta$-dynamics in simple liquids. Section 4 presents some MCT results for the final $\alpha$-relaxation in the liquid state. There, non-universal and wave vector dependent results are obtained. Results from probability theory shed light on the contents of the MCT description of the $\alpha$-process.

\section{Glass transition singularities of the idealized MCT}

The MCT of simple liquids is based on equations of motion for density fluctuation functions like the coherent intermediate scattering function $\Phi_{q}(t)=\frac{1}{N S_{q}}\left\langle\varrho_{-q}(t) \varrho_{\vec{q}}\right\rangle$, which is normalized to unity $\Phi_{q}(t=0)=1 . \Phi_{q}(t)$ and the incoherent intermediate scattering function $\Phi_{q}^{s}(t)=\left\langle e^{i \vec{q}\left(\vec{r}^{s}(t)-\vec{r}^{\prime}(0)\right)}\right\rangle$ are well defined functions of linear response theory and can be measured by neutron or light scattering experiments.

The dynamics of both correlators is connected to two memory functions $M_{q}^{l}(t)$ and $M_{q}^{s}(t)$ which provide simpler starting points for approximations $[1,6]$ :

$$
\partial_{t}^{2} \Phi_{q}(t)+\Omega_{q}^{2}\left\{\Phi_{q}(t)+\int_{0}^{t} d t^{\prime} M_{q}^{l}\left(t-t^{\prime}\right) \dot{\Phi}_{q}\left(t^{\prime}\right)\right\}=0
$$




$$
\partial_{t}^{2} \Phi_{q}^{s}(t)+v^{2}\left\{q^{2} \Phi_{q}^{s}(t)+\int_{0}^{t} d t^{\prime} M_{q}^{s}\left(t-t^{\prime}\right) \dot{\Phi}_{q}^{s}\left(t^{\prime}\right)\right\}=0
$$

where $v^{2}=\frac{k T}{m}$ and $\Omega_{q}^{2}=\frac{v^{2} q^{2}}{S_{q}} . M_{q}^{l}(t)$ and $M_{q}^{s}(t)$ are retarded damping functions.

Let us at first consider an approximation which neglects memory effects in the damping functions: $M_{q}^{l(0)}(t)=2 \Gamma_{q} \delta(t)$ and $M_{q}^{s(0)}(t)=2 \Gamma_{q}^{s} \delta(t)$. Equations (1) in this case generally describe damped harmonic oscillations. The coherent density fluctuations for small wave vectors $q$ lead to propagating sound waves with $\Phi_{q}^{(0)}(t)=\left(\cos \Omega_{q} t+\left(\Omega_{q} \Gamma_{q} / 2\right) \sin \Omega_{q} t\right) e^{-\gamma q^{2} t}$, with $\gamma=\Gamma_{0} v^{2} / 2 S_{0}$. The incoherent density fluctuations for small wave vectors show diffusive behavior: $\Phi_{q}^{s(0)}(t)=e^{-D^{s} q^{2} t}$, with $D^{s}=1 / \Gamma_{0}^{s}$. In hydrodynamics, the separation of time scales, expressed in the assumptions for $M_{q}^{l(0)}$ and $M_{q}^{s(0)}$, is justified by considering the hydrodynamic slow variables for $q \rightarrow 0$. The neglect of temporal correlations in the memory functions, however, in general, is not possible in liquids at high densities due to the glass transition singularity $[9,10]$. This is elaborated by the MCT. In this context it has to be mentioned that temperature fluctuations, which would show up in $\Phi_{q}(t)$, are neglected throughout this paper for the sake of simplicity.

In liquids of low densities, generalized transport theories allow to calculate contributions to $M^{l}$ and $M^{s}$ due to uncorrelated binary collisions of particles. For hard spheres a generalized Enskog theory provides explicit expressions for $M^{l}$ and $M^{s}$ due to binary collisions, see [11] and references therein. Let us denote these well understood contributions to $M^{l}$ and $M^{s}$ by $M^{i(B C)}$ and $M^{s(B C)}$ with some as yet unknown remainders $m_{q}(t)$ and $m_{q}^{s}(t)$ :

$$
\begin{aligned}
& M_{q}^{l}(t)=M_{q}^{l(B C)}(t)+m_{q}(t), \\
& M_{q}^{s}(t)=M_{q}^{s(B C)}(t)+m_{q}^{s}(t) .
\end{aligned}
$$

The approximations of the idealized MCT treating repeated and correlated collisions of particles which lead to back-flow and cage effect are described in the article of Götze and Sjögren in this volume. For the remainder of the memory functions the $\mathrm{MCT}$ formulates the following approximations:

$$
\begin{aligned}
& m_{q}(t)=\frac{1}{2} \int \frac{d^{3} k}{(2 \pi)^{3}} V(\vec{q}, \vec{k}) \Phi_{k}(t) \Phi_{|\vec{q}-\vec{k}|}(t), \text { with } \\
& V(\vec{q}, \vec{k})=n S_{q} S_{k} S_{|\vec{q}-\vec{k}|}\left\{\frac{\vec{q}}{q}\left[\vec{k} c_{k}+(\vec{q}-\vec{k}) c_{|\vec{q}-\vec{k}|}\right]\right\}^{2} / q^{2}
\end{aligned}
$$




$$
\begin{gathered}
m_{q}^{s}(t)=\int \frac{d^{3} k}{(2 \pi)^{3}} V^{s}(\vec{q} \vec{k}) \Phi_{k}(t) \Phi_{|\vec{q}-\vec{k}|}^{s}(t) \quad, \quad \text { with } \\
V^{s}(\vec{q}, \vec{k})=n\left(\frac{\vec{q} \vec{k}}{q}\right)^{2} c_{k}^{2} S_{k} .
\end{gathered}
$$

In this article, the results of equations (1-3) for the dynamics of hard spheres shall be discussed. If the known explicit expressions of $M^{l(B C)}$ and $M^{s(B C)}$ are used, equations (1) to (3) present a fully determined closed set of equations of motion for the correlation functions.

In order to demonstrate the role of the cage effect, let us at first consider an oversimplified model which neglects binary collisions completely, i.e.:

$$
M_{q}^{l}(t)=m_{q}(t) \quad \text { and } \quad M_{q}^{s}(t)=m_{q}^{s}(t) .
$$

The model now consists of the closed set of equations (1), (3) and (4) which are solved for different packing fractions $\varphi$. The long time decay of $\Phi_{q}^{s}(t)$ is still diffusive for small wave vectors and, from the numerical solutions, one can read off the long time self diffusion coefficient $D^{s}(\varphi)$. In Fig. 1 one notices: whereas $D^{s}$ only changes by a factor of 50 in between $\varphi=0.10$ and $\varphi=0.45, D^{s}$ decreases drastically in the small interval $0.45 \leq \varphi \leq \varphi_{c}$, where $\varphi_{c} \approx 0.525$. This allows to identify a first scaling time $\tau(\varphi)$ which is expected from the asymptotic analysis of the equations (1-4) $[3,4]$. For packing fractions close to the critical value $\varphi_{c}$ transport coefficients are determined by $\tau$; in this example $D^{s}=\hat{D}^{s} / \tau$, where $\hat{D}^{s} \approx 0.017 d^{2} . \tau(\varphi)$ diverges for $\varphi \rightarrow \varphi_{c}$ according to:

$\tau=t_{0} B^{-1 / b}|\sigma|^{-\gamma}$, with $\sigma \doteq C \frac{\varphi-\varphi_{c}}{\varphi_{c}}$, and $\gamma \approx 2.62, B^{-1 / b} \approx 0.98, C \approx 1.2-1.4$.

This result follows from the observation that self consistent MCT equations like (1-4) contain bifurcation singularities which lead to power law behaviors as denoted in (5) $[12,9,10]$. The numerical values entering (5), for example the exponent $\gamma=2.62$, are material dependent [4]. (5) denotes the values specific for a hard sphere liquid $[13,14]$. The time scale $t_{0}$ characterizes the transient dynamics and will be determined below.

Without repeating the analysis, let us state that close to the critical packing fraction the full equations of motion (1-3) simplify. The inertia term and the binary collison part in (1) become negligible [3]. The dynamics for long times and densities around $\varphi_{c}$ are described by equations (3) and: 


$$
\begin{gathered}
\Phi_{q}(t)+\int_{0}^{t} d t^{\prime} m_{q}\left(t-t^{\prime}\right) \dot{\Phi}_{q}\left(t^{\prime}\right)=0 \\
q^{2} \Phi_{q}^{s}(t)+\int_{0}^{t} d t^{\prime} m_{q}^{s}\left(t-t^{\prime}\right) \dot{\Phi}_{q}^{s}\left(t^{\prime}\right)=0 \quad \text { for } \varphi \rightarrow \varphi_{c} \quad \text { and } t \gg t_{\text {micro }} .
\end{gathered}
$$

From the asymptotic analysis moreover it follows that any further contribution to the memory functions like $M^{l(B C)}$ and $M^{s(B C)}$ which vary on microscopic time scales drop out off the long time dynamics. I.e. as long as microscopic time scales defined in analogy to

$$
t_{\text {micro }}=\int_{0}^{\infty} d t M^{l(B C)}(t) / M^{l(B C)}(t=0),
$$

stay finite for $\varphi \approx \varphi_{c}$ the equations $(6)$ will not change with $t_{\text {micro }}$ denoting the short time cut off [3].

In equations (3) and (6) one notices no inherent time scale; any solution $\Phi_{q}(t)$ gives infinitely many solutions $\Phi_{q}(t \cdot x)$ shifted in time. Therefore in formula $(5)$ the time scale $t_{0}$ cannot be obtained from the asymptotic analysis. It is determined by the microscopic dynamics and has to be found from matching to the transient dynamics. From Fig. 1 one obtains $t_{0} \approx 0.04 d / v$ which, if units appropriate for Argon are used, corresponds to $t_{0} \approx 1.510^{-12} \mathrm{sec}$. The asymptotic results of the MCT to be discussed below describe the power law divergence of $\tau(\varphi)$ in (5). Of course, the neglect of for example the well known binary collision contributions $M^{l(B C)}$ and $M^{s(B C)}$ falsifies the values of $\tau$ as long as $\tau$ is still comparable to $t_{\text {micro. }}$. The binary collisions would also influence the value of $t_{0}$.

Some direct consequences of the result that the long time dynamics of a dense liquid is described by equations (3) and (6) stem from the fact that these equations are fully determined by $S_{q}$. First, because of the prominent peak in $S_{q}\left(\varphi_{c}\right)$, which dominates the vertices in (3) and is rather universal for general simple liquids, one expects that the observed singularity exists in various liquids [4]. Second, because of the identical equilibrium structure displayed by hard spheres in vacuum and by hard spheres in a solvent, one deduces that both liquids display the same asymptotic dynamics close to $\varphi_{c}$. Especially an "atomic liquid of hard spheres in vacuum" and a "system of colloidal hard spheres dispersed in some solvent" should possess the identical critical density $\varphi_{c}$, the same exponents like $\gamma$ and should differ only in the value of $t_{0}$ in (5) [15].

Let us therefore also discuss the full equations of motion for density fuctuations of colloidal particles in some solvent with viscosity $\eta_{0}[2]$. 


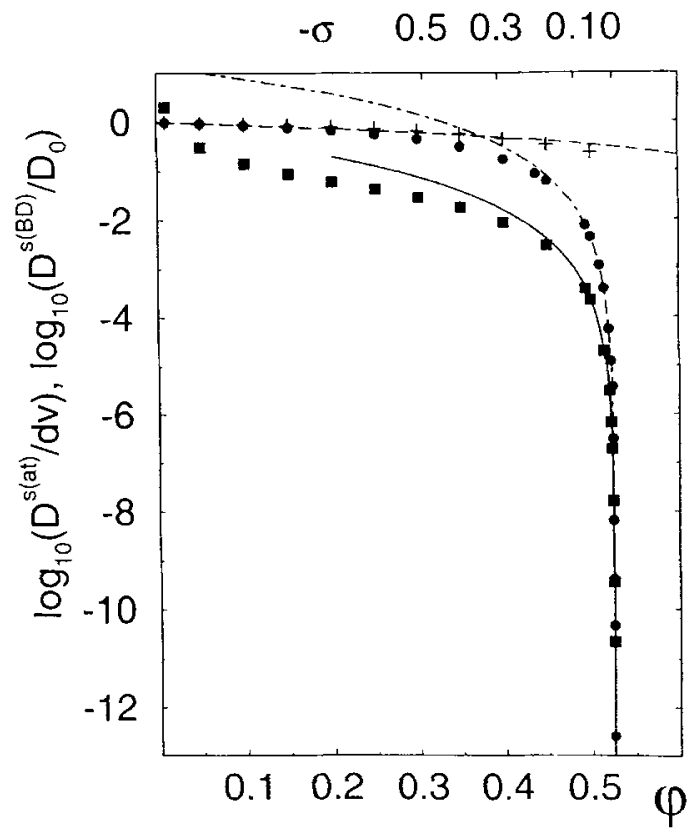

Fig. 1 Self diffusion coefficients $D^{s(a t)} / d v$ (squares) as a function of packing fraction $\varphi$ in an atomic hard sphere liquid described by $(1,3,4)$. The solid line is the asymptote (5) with $t_{0}=0.04 d / v$. The circles display the self diffusion coefficients $D^{s(B P)} / D_{0}$ of a system of interacting Brownian particles described by $(8,3,10)$. A chain curve shows the asymptote (5) with $t_{0}=2.210^{-3} d^{2} / D_{0} . \sigma$-values are indicated at the top of the figure. The perturbative calculation for $D^{s(B P)}$ using formula (11) is denoted by the crosses. The dashed line gives the low density result from (3) $D^{s} / D_{0}=1-4 / 3 \varphi$.

$$
\begin{gathered}
\partial_{t} \Phi_{q}(t)+D_{q} q^{2}\left\{\Phi_{q}(t)+\int_{0}^{t} d t^{\prime} \tilde{M}_{q}^{l}\left(t-t^{\prime}\right) \dot{\Phi}_{q}\left(t^{\prime}\right)\right\}=0 \\
\partial_{t} \Phi_{q}^{s}(t)+D_{0}\left\{q^{2}, \Phi_{q}^{s}(t)+\int_{0}^{t} d t^{\prime} \tilde{M}_{q}^{s}\left(t-t^{\prime}\right) \dot{\Phi}_{q}^{s}\left(t^{\prime}\right)\right\}=0
\end{gathered}
$$

where $D_{0}=\frac{k T}{3 \pi \eta_{0} d}$ and $D_{q}=\frac{D_{0}}{S_{q}}$.

The above result (6) of the MCT derived for "atomic" hard spheres described by equation (1) shows that the memory functions $\tilde{M}^{l}$ and $\tilde{M}^{s}$ close to $\varphi_{c}$ can be written as: 


$$
\begin{aligned}
& \tilde{M}_{q}^{l}(t)=M_{q}^{l(B D)}(t)+m_{q}(t), \\
& \tilde{M}_{q}^{s}(t)=M_{q}^{s(B D)}(t)+m_{q}^{s}(t),
\end{aligned}
$$

where $m_{q}(t)$ and $m_{q}^{s}(t)$ are given in formulae (3) and $M_{q}^{l(B D)}$ and $M_{q}^{s(B D)}$ are memory functions which relax on finite time scales for $\varphi$ close to $\varphi_{c}$. The damping functions $M_{q}^{(B D)}$ in principle may contain the so called hydrodynamic interactions which are peculiar to colloidal suspensions. It is not surprising that equations (8) and (9) or versions very similar to them have been used to describe the dynamics of colloidal systems at intermediate densities although without hydrodynamic interactions $[16$, 17]. As stated, the MCT results (6) and (5) will again hold, if the asymptotic time scales like $\tau(\varphi)$ become large compared to the microscopic time scale $t_{\text {micro }}$ resulting from the $M^{(B D)}$ in (9). This is also exemplified in Fig. 1, where the self diffusion coefficients $D^{s}(\varphi)$ are plotted as obtained from the numerical solutions of equations (3) and (8) where the short time memory functions $M_{q}^{l(B D)}$, and $M_{q}^{s(B D)}$ were neglected, i.e.:

$$
\tilde{M}_{q}^{l}(t)=m_{q}(t) \quad \text { and } \quad \tilde{M}_{q}^{s}(t)=m_{q}^{s}(t)
$$

Again, for $\varphi$ close to $\varphi_{c}$ the asymptotic result $(5)$ is found with $t_{0} \approx 2.210^{-3} d^{2} / D_{0}$ this time. In realistic units [2], however, this value, $t_{0} \approx 10^{-5} \mathrm{sec}$, is about 7 decades larger than the $t_{0}$ of the atomic hard sphere system.

If one had neglected the memory functions $\tilde{M}_{q}^{l}$, and $\tilde{M}_{q}^{s}$ in (8), one would have obtained the known diffusive results of Brownian motion [2]: $\Phi_{q}^{(0)}(t)=e^{-D_{q} q^{2} t}$ and $\Phi_{q}^{s(0)}(t)=e^{-D_{0} q^{2} t}$. In some contexts MCT equations have been obtained by perturbative calculations which lead to the result, that the memory functions have to be calculated as integrals over such zeroth order results for the correlators [16]; i.e.:

$$
\begin{aligned}
& m_{q}^{(1)}(t)=\frac{1}{2} \int \frac{d^{3} k}{(2 \pi)^{3}} V(\vec{q}, \vec{k}) \Phi_{k}^{(0)}(t) \Phi_{|\vec{q}-\vec{k}|}^{(0)}(t) \\
& m_{q}^{s(1)}(t)=\int \frac{d^{3} k}{(2 \pi)^{3}} V^{s}(\vec{q} \vec{k}) \Phi_{k}^{(0)}(t) \Phi_{\{\vec{q}-\vec{k} \mid}^{s(0)}(t)
\end{aligned}
$$

One may consider calculating the memory functions from equations (11) and using them in equations (8) in order to obtain the first order approximations $\Phi_{q}^{(1)}(t)$ and $\Phi_{q}^{s(1)}(t)$ as a perturbative treatment of the self-consistency problem in (8), (3) and (10). In Fig. 1 one observes that perturbative and self-consistent results coincide for low densities. However the perturbative calculation fails already at $\varphi \approx 0.20$ long 
before the influence of the glass transition singularity at $\varphi_{\mathrm{c}}$ sets in around $\varphi \approx 0.45$. From these results for the diffusion coefficients, one may conclude that the full MCT equations of motion contain a self consistency problem which at increasing densities leads to a critical slowing down of the dynamics. As will be argued below using further results from the calculations for a hard sphere liquid, this self consistency problem can be viewed as a mathematical description of the cage effect mentioned in the introduction. As a side remark, it may be noted that the MCT result for vanishing density $D^{\mathfrak{s}} / D_{0}=1-\frac{4}{3} \varphi+\mathcal{O}\left(\varphi^{2}\right)[18]$ is rather close to the correct result for interacting Brownian particles $D^{s} / D_{0}=1-2 \varphi+\mathcal{O}\left(\varphi^{2}\right)$ [19].

For colloidal systems, the self diffusion coefficient $D^{s}$ is connected to the integral over all times of the memory function $\tilde{M}_{q=0}^{s}(t)$ via:

$$
D_{0} / D^{s}=1+\int_{0}^{\infty} d t \tilde{M}_{q=0}^{s}(t) .
$$

Considerably more information than from the value of this integral can be obtained from the time dependence of various correlation functions. Fig. 2 depicts the coherent density fluctuation functions $\Phi_{q}(t)$ of the colloidal system for different densities and at two wave vectors, $q_{0}=4.4 / a$ the position of the peak in $S_{q}$ and $q_{1} \approx q_{0} / 2$. Again the equations $(8,3,10)$ were solved numerically. For low densities, the expected almost exponentional decay is observed. At higher densities, however, one clearly notices the evolution of a two step decay process. $\Phi_{q}(t)$ decays slowly around some $q$-dependent plateau value denoted by $f_{q}^{c}$ and relaxes to zero on a much longer time scale $\tau_{q}(\varphi)$. This $\tau_{q}(\varphi)$ is found to be proportional to the $\tau(\varphi)$ in equation (5); $\tau_{q}(\varphi)=\hat{\tau}_{q} \tau(\varphi)$, with $\hat{\tau}_{q 0} \approx 1.74$ and $\hat{\tau}_{q_{1}} \approx 0.26$. Another time scale denoted by $t_{\sigma}$ can be read off for example from the time where $\Phi_{q}(t)$ crosses the plateau value $f_{q}^{c}$ (which can be determined independently): one finds a $q$-independent time scale $t_{\beta}^{-}$, where $\Phi_{q}\left(t_{\beta}^{-}\right)=f_{q}^{c}$ and $t_{\beta}^{-}=0.6 t_{\sigma}$, with:

$$
t_{\sigma}=t_{0}|\sigma|^{-\frac{1}{2 a}}, \quad \text { with } a=0.297,
$$

where the same $\sigma$ and $t_{0}$ enter as in equation (5). The existence of a second time scale $t_{\sigma}$ besides $\tau$ is a rather unexpected finding of the MCT $[9,10]$ which cannot be discovered by looking at transport coefficients like $D^{s}$ in (12) only. Even more strikingly, above the mentioned critical packing fraction $\varphi_{c}$, all correlation functions $\Phi_{q}(t)$ or $\Phi_{q}^{s}(t)$ do not decay to zero but arrest on finite values $f_{q}(\varphi)$ and $f_{q}^{s}(\varphi)$ respectively for $t \rightarrow \infty$. 

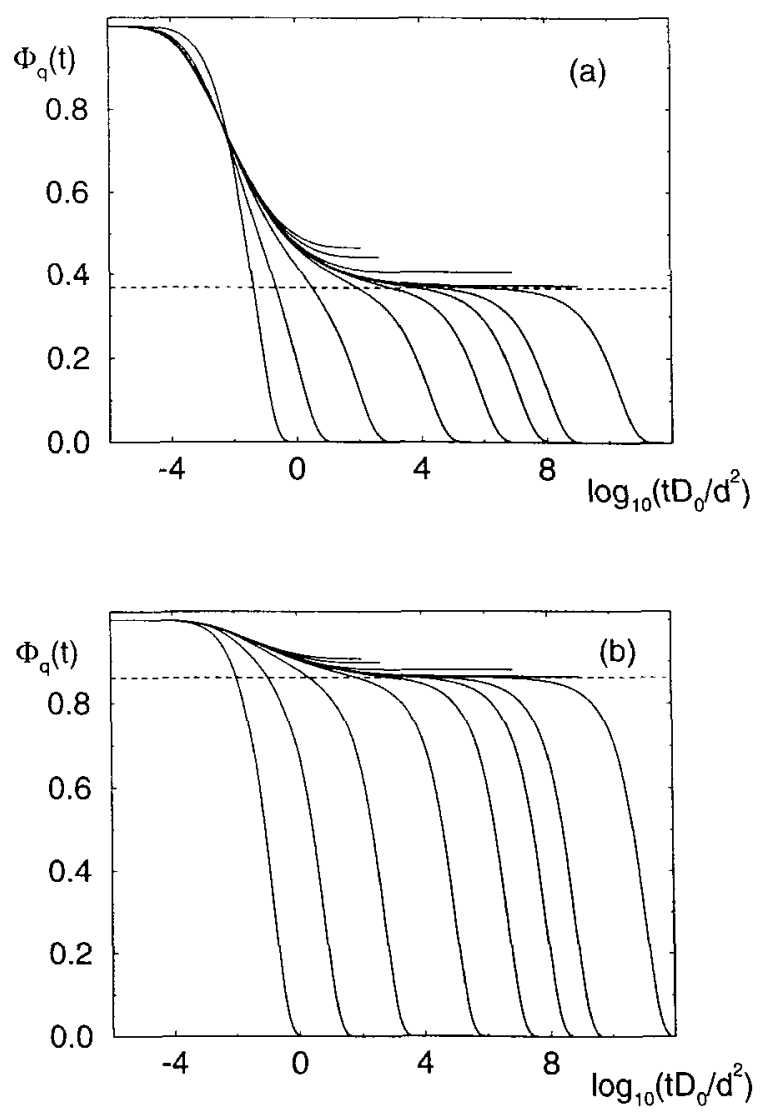

Fig. 2 Normalized coherent density correlators $\Phi_{q}(t)$ at two wave vectors, $q_{1}$ in (a) and $q_{0}$ in (b), for different densities; $q_{0}=4.4 / a$ is the position of the peak in $S_{q}$ and $q_{1}=2.1 / a \approx q_{0} / 2$, where $a=\sqrt[3]{(3 / 4 \pi n)}$ is the averaged interparticle distance. The dotted lines denotes the critical non-ergodicity parameter $f_{q_{0}}^{c} \approx 0.861$ and $f_{q_{1}}^{c} \approx 0.368$. From left to right the packing fractions $\varphi$ and separations $\varepsilon=(\varphi-$ $\left.\varphi_{\mathrm{c}}\right) / \varphi_{\mathrm{c}}$ equal: $(0.40,-0.24),\left(0.50,-4.710^{-2}\right),\left(0.520,-8.910^{-3}\right),\left(0.524,-1.210^{-3}\right)$, $\left(0.5245,-2.910^{-4}\right),\left(0.5246,-1.010^{-4}\right),\left(0.52463,-4.410^{-5}\right),\left(0.52465,-5.810^{-6}\right)$, $\left(0.52466,1.310^{-5}\right),\left(0.525,6.610^{-4}\right),\left(0.526,2.610^{-3}\right),\left(0.527,4.510^{-3}\right)$. 
Fig. 3 demonstrates that the rise of the long time values $f_{q}(\varphi)$ shows singular behavior. As familiar from bifurcation problems, $f_{q}$ rises with infinite slope at the critical point [20]:

$$
f_{q}(\varphi)=f_{q}^{c}+h_{q} C^{\prime} \sqrt{\frac{\varphi-\varphi_{c}}{\varphi_{c}}}+\mathcal{O}\left(\varphi-\varphi_{c}\right), \quad \text { with } \quad C^{\prime}=2.26 \quad \text { for } \varphi>\varphi_{c}
$$

Whereas the static structure factor in Fig. $3 \mathrm{~b}$ only changes smoothly around $\varphi_{\mathrm{c}}$, the non-ergodicity parameter $f_{q}$ changes drastically as can be seen in Fig. 3a. Fig. $3 a$ also gives some indication for the range of applicability of the asymptotic results which will be discussed in the later sections. The asymptotic result (14) holds for ca. $\left(\varphi-\varphi_{c}\right) / \varphi_{c} \leq 0.03$ for wave vectors in a range around $q_{0}$ the position of the peak in $S_{q}[21]$.

In Fig. 2 , if one reads off a time scaie $t_{\beta}^{+}$where the decay of the $\Phi_{q}(t)$ crosses over to the rising plateau $f_{q}(\varphi)$, one notices that this time is proportional to the time scale $t_{\sigma}$ given in (13) and decreases with increasing density.

After this cursory glimpse on the dynamical singularity at $\varphi_{c}$, some remarks about the value of (numerical) solutions of the full set of MCT equations $(1,2,3)$ or $(3,8,9)$ might be appropriate.

First, the comparison in Fig. 1 shows that the perturbative calculation for $D^{s}$ clearly does not capture the feed-back mechanism contained in the full equations.

Second, equations (3) and (6) valid for $\varphi \rightarrow \varphi_{c}$ and $t \gg t_{\text {micro }}$ capture this feed-back mechanism. Two scaling laws, the so called $\beta$-and $\alpha$-relaxation scaling laws capture the essence of these mathematical non-linearities, see [3] for more information. In order to describe the dynamics of a hard sphere liquid for all densities and for shorter times as well, reliable approximations for the microscopic memory functions like $M^{(B C)}$ and $M^{(B D)}$ are necessary. For the long time dynamics, however, the solutions of the two asymptotic scaling laws contain more information because the feed-back mechanism can be studied without system specific microscopic corrections. Third, comparisons with experiments on hard sphere liquids show that the approximations (3) lead to a critical packing fraction $\varphi_{c} \approx 0.525$ which is roughly $10 \%$ lower than the experimental value $\varphi_{c}^{E x p} \approx 0.58$ [22]. The difficulty of calculating the values of critical densities or temperatures is known from such calculations in the field of phase transitions. In order to make meaningful comparisons between the theoretical results close to the singularity and the experimental data, the separation from the critical density

$$
\varepsilon=\frac{\varphi-\varphi_{c}}{\varphi_{c}}
$$




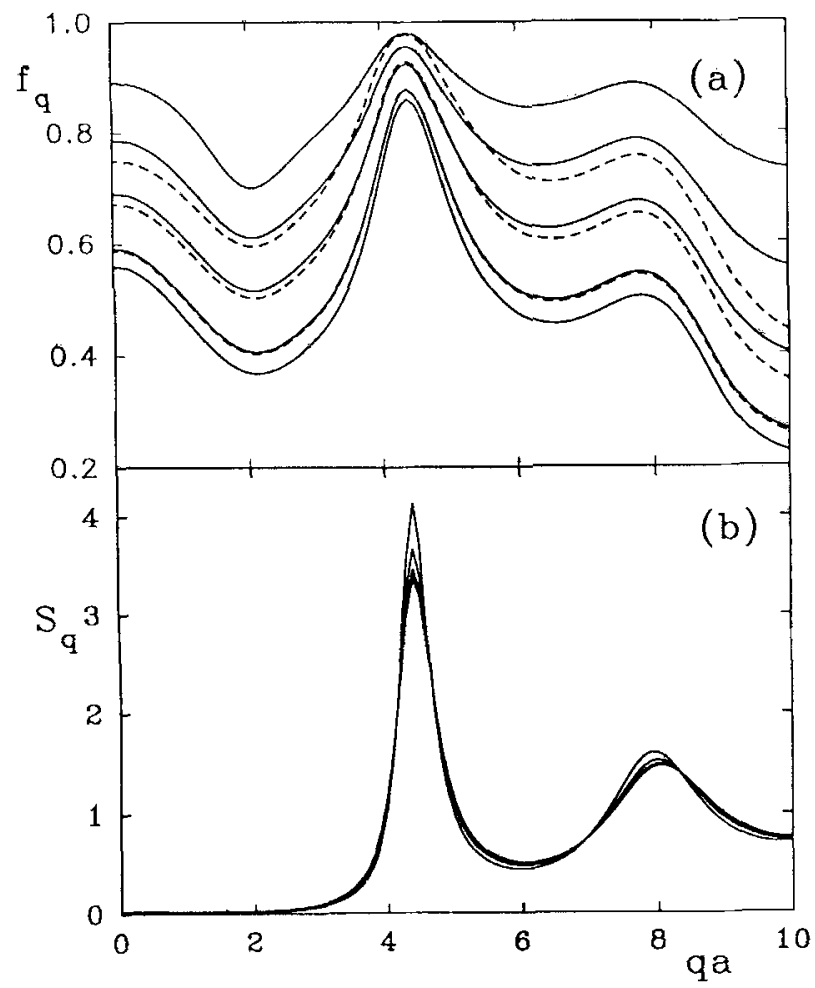

Fig. 3 In (a) the non-ergodicity parameters $f_{q}$ (solid lines) for four different separations $\varepsilon=\left(\varphi-\varphi_{c}\right) / \varphi_{c}$ are shown as function of $q$ using the Verlet-Weis structure factor from Fig. (b) as input; $\varepsilon=0,7.010^{-4}, 0.010,0.029$ and 0.067 ; from [21]. $a=\sqrt[3]{(3 / 4 \pi n)}$ is the averaged interparticle distance. The dashed lines show the asymptotic law $f_{q}=f_{q}^{c}+2.26 h_{q} \sqrt{\varepsilon}$ for the same separations $\varepsilon$. The critical packing fraction is $\varphi_{c}=0.525$.

has to be used instead of the density itself. Allowing for this use of $\varepsilon$ and the unknown time scale $t_{0}$ equations (3) and (6) or the asymptotic scaling laws discussed in the next sections provide a description of the long time dynamics which is fully determined from the static structure factor $S_{q}$. For example, the ratio $D^{s} / D_{0}$ will rather coincide for different systems, if the $S_{q}$ and consequently the $\sigma$ are similar. This corresponds well to a recent finding that, at the density of freezing, $\varphi_{F}$, the 
self diffusion coefficients of various colloidal suspensions take an universal value $D^{s} / D_{0} \approx 0.10$ [23]. The analysis of data from colloidal hard spheres using the MCT shows that, at $\varphi_{F}=0.494$, the asymptotic results are at their range of validity [22]; $\sigma \approx-0.20$ is found at $\varphi_{F}$. The $D^{s}$ resulting from equations $(3,8,10)$ for $\sigma=-0.20$, i.e. for $\varphi=0.437$, equals $D^{s} / D_{0} \approx 0.085$.

Fourth, the neglect of further transport mechanism which lead to a decay of the correlation functions for $\varphi>\varphi_{c}$ does not invalidate the idealized MCT results even in systems where hopping processes are present. From the extended MCT, the range of validity of the idealized MCT can be determined [7].

\section{The first scaling law region}

The dynamics of the MCT equations can be simplified considerably for $\Phi_{q}(t)$ close to the plateau value $f_{q}^{c}$ and for small differences of the vertices $V(\vec{q}, \vec{k})$ in (3) from their values at the critical density [3]. One obtains a separation of spatial and temporal dependences in the limit $\varphi \rightarrow \varphi_{c}$ and $t_{\sigma} \rightarrow \infty$ [20]:

$$
\Phi_{q}(t)=f_{q}^{c}+h_{q} \sqrt{\sigma} g_{ \pm}^{\lambda}\left(t / t_{\sigma}\right)+\mathcal{O}(\sigma) \quad \sigma \gtrless 0 .
$$

A similar result holds for $\Phi_{q}^{s}(t)$, where $f_{q}^{c}$ and $h_{q}$ have to be replaced by $f_{q}^{s}$ and $h_{q}^{s}$ respectively. The $\beta$-correlator $g_{ \pm}^{\lambda}(x)$ obeys a scaling equation specified by the so called exponent parameter $\lambda$. All quantities in (15) except for the time $t_{0}$ can be calculated in simple liquids from the static structure factor $S_{q}$. One finds the result for the critical packing fraction denoted in $(5)[10,13,14]$ and the exponent parameter $\lambda[13,14]$ :

$$
\lambda=0.766 .
$$

Except for these material dependent values the $\beta$-correlator $g_{ \pm}^{\lambda}(x)$ characterizes the type of singularity at $\varphi_{c}\left(\mathrm{~A}_{2}\right.$-Whitney fold bifurcation in this case) and may for example be studied in simplified schematic MCT models, as well. At present, the value of the exponent parameter $\lambda$, which follows from a complicated integral over all wave vectors [20], cannot be connected to specific material properties.

The contents of the scaling law (15) is displayed in Fig. 4. There, the $\beta$-correlator for the case of liquid and glass is obtained from the curves $\quad 2$. 2 by plotting $\left(\Phi_{q}(t)-f_{q}^{c}\right) / h_{q} \sqrt{\sigma}$ for different wave vectors and densities. nu used wave vector, one observes that for $\sigma \rightarrow 0$ the replotted curves follow the $\beta$-correlator over an 

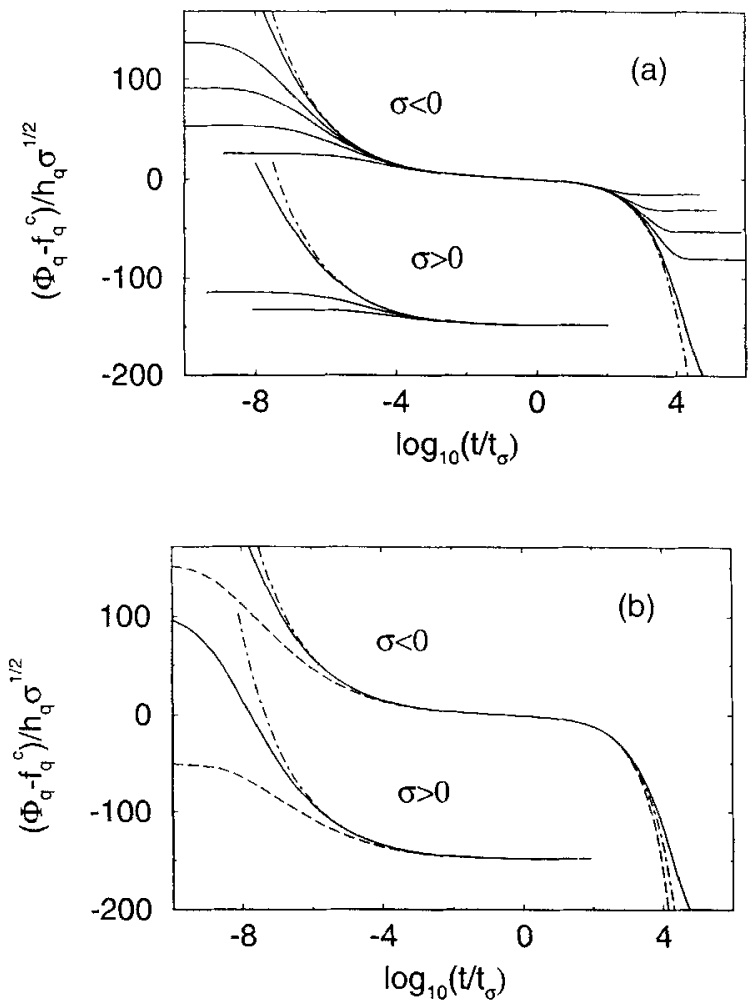

Fig. $4 \beta$-scaling plots of $\left(\Phi_{q}(t)-f_{q}^{c}\right) / h_{q} \sqrt{\sigma}$, where the correlators $\Phi_{q}(t)$ are taken from Fig. 2. The chain curves denote the $\beta$-correlators $g_{ \pm}$. The glass curves are shifted vertically by -150 for clarity. The $h_{q}$ are $h_{q_{0}} \approx 0.30$ and $h_{q_{1}} \approx 0.59$.

In (a), the 5 packing fractions $\varphi$ below and closest to $\varphi_{c}$ and the 3 packing fractions $\varphi$ above and closest to $\varphi_{c}$ are plotted for the wave vector $q_{1}=2.2 / a$ versus the scaled time $t=t / t_{\sigma}$, with $t_{\sigma}$ from (5).

In (b), the correlators for the two wave vectors $q_{0}=4.4 / a$ (broken lines) and $q_{1}=$ $2.1 / a$ (solid lines) are compared at the packing fractions closest to the critical density. 
expanding window in time. For fixed density, replotted curves for different wave vectors overlap in a time window, thereby, tracing out the $\beta$-correlator. The wave vector dependence of the corrections to (15) is apparent in Fig. 4.

The wave vector dependent non-universal quantities $f_{q}^{c}$ and $h_{q}$ characterize the transport mechanism which leads to the appearance of the power law decay in $g_{ \pm}^{\lambda}[3]$. At the critical packing fraction $\varphi_{c}$ the correlation functions arrest for long times at their non-ergodicity values $f_{q}^{c}$. The Fourier transform $F(r)=1+\frac{1}{n} \int \frac{d^{3} q}{(2 \pi)^{3}} e^{i \overrightarrow{q r}} S_{q} f_{q}^{c}$ describes the frozen in structure of the glass at the critical density $\varphi_{c} . F(r)$ may be compared with other descriptions of the structure of an amorphous solid. A configuration of randomly packed hard spheres leads to an irregular lattice with a site-site pair correlation function $g_{L}\left(\vec{R}-\vec{R}^{\prime}\right)$ obtainable for example by computer studies [24]. At the density at which the transition from an amorphous solid to a liquid takes place, one can expect the particles to be distributed around their sites $\vec{R}_{i}$ with some probability distribution $\phi(\vec{r}-\vec{R})$ which is characterized by the Lindemann ratio. The Lindemann criterion for the melting of a crystal states that melting takes place if the average displacement of an atom from its lattice site equals $\sqrt{\left\langle\Delta r^{2}\right\rangle}=$ $0.15 d$ [1]. In Fig. 5 from [13] the MCT result for $F(r)$ is compared to the structure one obtains from uncorrelated Gaussian fluctuations with mean $\sqrt{\left\langle\Delta r^{2}\right\rangle}=0.11 d$ around the random closed packed structure simulated on a computer [24]. This comparison demonstrates that the MCT calculates for the glassy structure of hard spheres a smeared out, randomly close packed structure.

The fluctuations of the particles around their sites can also be read off from the localization length of a single particle at $\varphi_{\mathrm{c}}$. The Lamb-Mössbauer-factor $f_{q}^{s}$ measures the localization of a particle in the glassy phase. It follows a Gaussian, $f_{q}^{s}=e^{-q^{2} r_{s}^{2}}$, rather closely with root mean square displacement $\sqrt{\left\langle\Delta r^{2}\right\rangle} \approx 0.10 d$ reproducing the Lindemann criterion $[10,14]$.

The reduction theorem (15) expresses that the frozen-in structures $f_{q}^{c}$ or $f_{q}^{s}$ become instable by fluctuations whose spatial dependence decouples from the temporal dependence. The spatial variation is given by the critical amplitudes $h_{q}$ or $h_{q}^{s}$. The Fourier transform $H(r)=\frac{1}{n} \int \frac{d^{3} q}{(2 \pi)^{3}} e^{i \vec{q} \vec{r}} S_{q} h_{q}$ tells on which distances density fluctuations relax during the $\beta$-process. In Fig. 6 , one recognizes that only rearrangements of the nearest and next to nearest neighbor shells occur in the $\beta$-process [13]. This justifies the claim of the idealized MCT to study the feed-back mechanism of the cage effect. The $\beta$-process results from the slow fluctuations of the cages formed by neighboring particles. The feed-back mechanism is captured in the mathemat- 


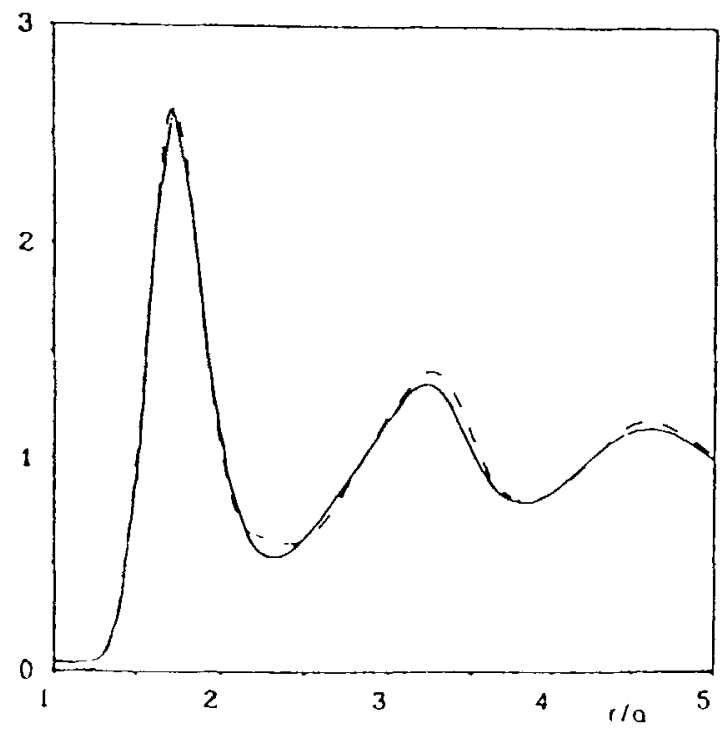

Fig. 5 Comparison of the glassy structure $F(r)$ (solid line) of the MCT at the critical packing fraction $\varphi_{c}$ with the smeared out random close packed structure (broken line) obtained from the computer study in [24] and using the Lindemann ratio; from [13]. The average distance equals $a \approx 0.62 d$.

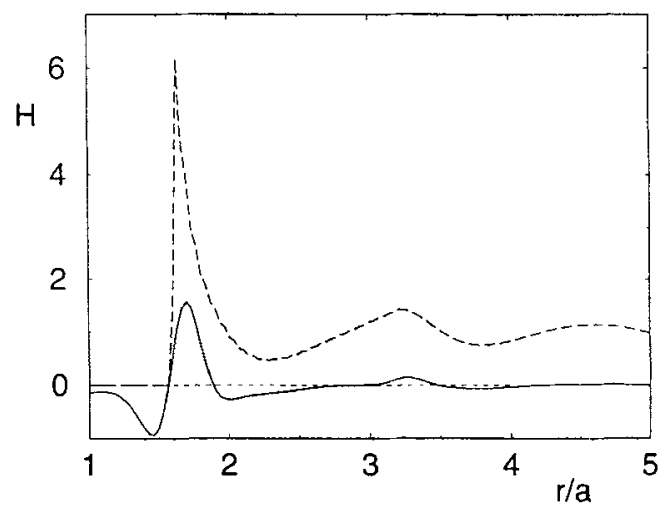

Fig. 6 The critical amplitude $H(r)$ (solid line) of the coherent density fluctuations is compared to the pair correlation function $g(r)$ (broken line); $\varphi=\varphi_{c}, a \approx 0.62 d$ [13]. 
ical non-linearities of the idealized MCT leading to divergent time scales like $t_{\sigma}$ and to the peculiar decoupling of the dynamics stated in (15). The result for $H(r)$ characterizes the $\beta$-process as localized dynamics. For simple liquids with smooth pair potentials or for mixtures of not too different particles qualitatively analogous results will be obtained as long as the static structure factors $S_{q}$ do not differ too much from the one of a hard sphere liquid.

\section{The second scaling law region}

In the liquid, i.e. for $\varphi<\varphi_{c}$, the $\beta$-correlator $g_{ \pm}^{\lambda}\left(t / t_{\sigma}\right)$ in (16) diverges for times long compared to the $\beta$-scaling time $t_{\sigma}$; this is described by the so called von Schweidler law $[25,20]$ :

$$
\lim _{t / t_{\sigma} \rightarrow \infty} \sqrt{\sigma} g_{ \pm}^{\lambda}\left(t / t_{\sigma}\right)=-\sqrt{\sigma} B\left(t / t_{\sigma}\right)^{b}=-(t / \tau)^{b} .
$$

The von Schweidler exponent $b$ is connected to the exponent parameter $\lambda$ and thereby to $S_{q}\left(\varphi_{c}\right) ; b=0.532$ in the hard sphere liquid $[13,14] .(17)$ is one of the central results of the MCT because it connects the two asymptotic dynamical processes. The von Schweidler power law behavior is shared by both scaling laws, the $\beta$-scaling law on time scale $t_{\sigma}$ and the $\alpha$-scaling law on scale $\tau$.

Close to $\varphi_{c}$ and on time scale $\tau$, the full MCT equations (1-3) are solved by a second set of master functions $\hat{\Phi}_{q}(\hat{t})$ which are independent of $\varphi$ [26]:

$\Phi_{q}(t)=\hat{\Phi}_{q}(t / \tau)+\mathcal{O}\left(\sigma X_{q}(t / \tau)\right) \quad$ for $\varphi-\varphi_{c} \rightarrow-0, \quad \tau \rightarrow \infty \quad$ and $t / \tau=$ fixed

A corresponding result holds for the incoherent density fluctuations $\Phi_{q}^{s}(t)$. The $\hat{\Phi}_{q}(\hat{t})$ pick up the von Schweidler divergence (17) as a short time behavior [25]:

$$
\hat{\Phi}_{q}(t / \tau)=f_{q}^{c}-h_{q}(t / \tau)^{b}+\mathcal{O}\left((t / \tau)^{2 b}\right)
$$

Equations (17) and (19) explicitly show how the von Schweidler law connects both scaling laws. The $\alpha$-relaxation amplitude $f_{q}^{c}$ and the critical amplitude $h_{q}$ in (19) agree with the quantities discussed in chapter 3 and may experimentally be measured using either (15) or (19). Equation (19) also shows that the $\alpha$-relaxation amplitudes $f_{q}^{c}$ or functions of them determine various dispersion relations. For example, the longitudinal sound velocity $c_{\infty}$ in the glass at $\varphi_{c}$ or in the liquid for frequencies which lie in the range $1 / \tau \ll \omega \ll 1 / t_{\sigma}$ follows: $c_{\infty}=c_{0} \sqrt{1 /\left(1-f_{0}^{c}\right)} \approx 1.51 c_{0}$. Here 
$c_{0}$ denotes the isothermal sound velocity of the liquid. Similarly the transversal sound velocity $c_{t}$ turns out to be $c_{t} \approx 0.64 c_{0}$ for the hard sphere liquid [14].

One notices in equation (18) that $\Phi_{q}(t)$, on the time scale $\tau$, in general depends on $\varphi$ not only via the strong increase of $\tau(\varphi)$. In lowest order in $\sigma \propto\left(\varphi-\varphi_{c}\right) / \varphi$, however, the time temperature superposition principle is obtained [3]. It states that the shape of the $\alpha$-relaxation curves is independent of $\varphi$ and given by the $\alpha$-master functions $\hat{\Phi}_{q}(\hat{t})$. The corrections to the $\alpha$-scaling law in (18) are of order $\sigma$. So they even are one order of $\sqrt{\sigma}$ smaller than the relative corrections to the $\beta$-scaling law in (15). From the discussion of the range of validity of the square root behavior of the non-ergodicity parameters one estimates that the $\alpha$-scaling law applies for $\varphi_{c}-\varphi$ $\leq 0.09$ roughly.

Fig. 7 exemplifies the $\alpha$-scaling law. The curves from Fig. 2 are shifted in time according to the $\alpha$-scaling time $\tau(\varphi)(5)$. The correlators for different densities follow the $q$-dependent $\alpha$-master functions for an increasing window in time upon an increase of the density, $\varphi \rightarrow \varphi_{c}$.

A first consequence may be drawn from the existence of one scaling time $\tau(\varphi)$ for the final $\alpha$-relaxation. Fluctuations on different length scales $1 / q$ or for different correlators relax on time scales $\tau_{q}(\varphi)$ whose density dependence agrees except for some constant prefactor $\hat{\tau}_{q}$, i.e. $\tau_{q}(\varphi)=\hat{\tau}_{q} \tau(\varphi)$ [4]. Density fluctuations on microscopic length scales $\approx 1 / q_{0}$ can be scaled using macroscopic transport coefficients like the shear viscosity $\eta_{s}=n k T \hat{\eta}_{s} \tau(\varphi)$, with $\hat{\eta}_{s}=5.83$ for hard spheres; please note the typing error in [14]. This also shows that the MCT predicts the Stokes-Einstein relation to hold quantitatively in a dense liquid of hard spheres for $\varphi<\varphi_{c}$ : the calculation gives $D^{s} \eta_{s}=\frac{k T}{6 \pi R}$, with $R \approx 0.53 d$ [14].

Such quantitative results of the MCT for the $\alpha$-process allow severe tests of the approximations underlying the idealized MCT. The equations for the $\alpha$-master functions explicitly depend on all details of the vertices $V^{c}(\vec{q}, \vec{k})$ and thereby via (3b) on the static structure factor $S_{q}$ evaluated at $\varphi_{c}[26]$ :

$$
\begin{aligned}
& \hat{\Phi}_{q}(\hat{t})=\hat{m}_{q}(\hat{t})-\frac{d}{d \hat{t}} \int_{0}^{\hat{t}} d t^{\prime} \hat{m}_{q}\left(\hat{t}-t^{\prime}\right) \hat{\Phi}_{q}\left(t^{\prime}\right), \\
& \hat{m}_{q}(\hat{t})=\frac{1}{2} \int \frac{d^{3} k}{(2 \pi)^{3}} V^{c}(\vec{q}, \vec{k}) \hat{\Phi}_{k}(\hat{t}) \hat{\Phi}_{|\vec{q}-\vec{k}|}(\hat{t}) .
\end{aligned}
$$

As Fig. 7 demonstrates, strong variations are obtained for density fluctuations with wave vectors $q$ around the peak position $q_{0}$. The relative relaxation times 


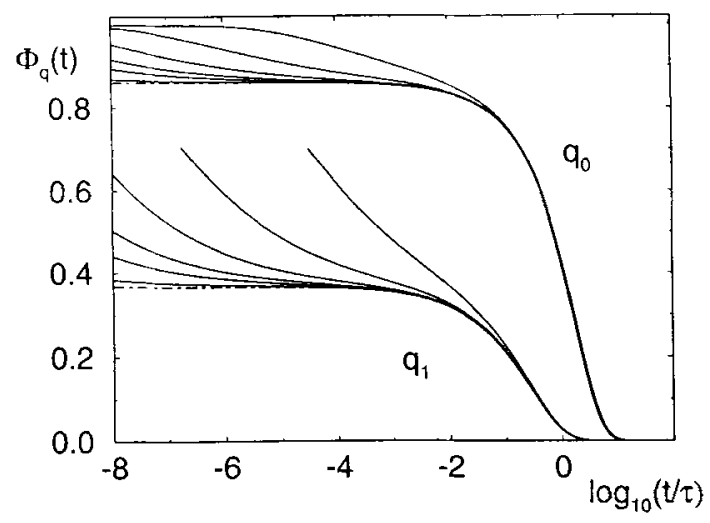

Fig. $7 \alpha$-scaling plots of the correlators $\Phi_{q}(t)$ from Fig. 2 for the six densities below and closest to $\varphi_{c}$. T The scaled time $\hat{t}=t / \tau$ is used, with $\tau$ taken from (5). The chain curves denote the $\alpha$-master functions.

$\hat{\tau}_{q}$ vary with $q$ up to a factor 6 . Also, the range of validity of the von Schweidler asymptote varies, as well as the non-exponentionality of the $\alpha$-master functions $\hat{\Phi}_{q}(\hat{t})$. Non-exponentional $\alpha$-decay, in general, results from the fractal von Schweidler short time asymptote (19) [20]. Let us study a typical $\alpha$-relaxation curve of the idealized MCT for simple liquids. Most conveniently this is done looking at a susceptibility spectrum. In Fig. 8 the spectrum of the longitudinal modulus $\omega m^{\prime \prime}(\omega)=\omega \int_{0}^{\infty} d t \cos (\omega t) m(t)$ clearly differs from the one of exponentional relaxation. The asymmetry of the $\alpha$-peak results from the stretching introduced by the von Schweidler law on the high frequency wing, $\chi^{\prime \prime}(\omega \tau \gg 1) \propto(\omega \tau)^{-b}$. Looking back on equations (15), (17) and (19) one may remember that the von Schweidler law was an analytical result valid for all correlators with exponent $b$ to be calculated from $S_{q}$.

Empirically, one observes that the $\alpha$-peaks $\hat{\Phi}_{q}(\hat{t})$ calculated for simple liquids can be fitted reasonably by the Kohlrausch function:

$$
\hat{\Phi}_{q}^{K}(\hat{t})=f_{q}^{K} e^{-\left(\hat{t} / \hat{\tau}_{q}\right)^{\beta_{q}}},
$$

with wave vector dependent amplitudes $f_{q}^{K}$, relaxation times $\hat{\tau}_{q}$ and stretching exponents $\beta_{q}$ [14]. In formula (21) $\hat{\tau}_{q}$ is the density independent prefactor introduced 


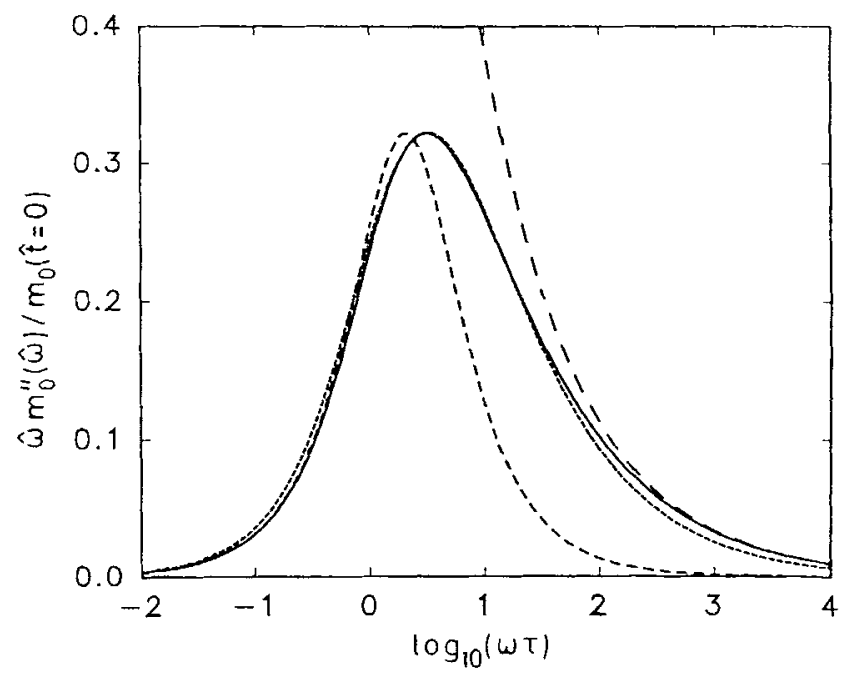

Fig. 8 Normalized spectrum of the longitudinal modulus $\hat{\omega} \hat{m}_{q=0}^{\prime \prime}(\hat{\omega})[14]$. The von Schweidler high-frequency asymptote (long dashes, $b=0.523$ ) and a fit to the lowfrequency wing assuming exponentional relaxation (short dashes) are included. A Kohlrausch fit with parameters $\hat{\tau}=0.25$ and $\beta=0.61$ is shown as dotted line.

above, which connects $\tau_{q}(\varphi)$ to $\tau(\varphi)$, i.e. $\tau_{q}(\varphi)=\hat{\tau}_{q} \tau(\varphi)$. In general the fits with the Kohlrausch function are fits of the quality displayed in Fig. 8. Systematic discrepancies at high frequencies due to $\beta_{q}>b$ can be observed. These deviations are not due to faster processes like the $\beta$-relaxation since the equations (20) describe the asymptotic $\alpha$-master functions only.

Figs. $9 \mathrm{a}$ and $9 \mathrm{~b}$ display the $q$-dependence of the Kohlrausch parameters fitted to the $\alpha$-master functions of the coherent density fluctuation functions [14]. Variations in phase with the non-ergodicity parameter $f_{q}^{c}$ and thereby with the structure factor $S_{q}$ demonstrate the close connection of these results to the underlying equilibrium structure. The relative relaxation time $\hat{\tau}_{q}$ changes by a factor of ca. 6 around $q_{0}$ and the stretching exponent $\beta_{\mathrm{q}}$ by $20 \%$. The maximum of $\hat{\tau}_{q}$ at $q_{0}$ is due to the slow break up of the spatial correlations on the length scale of the average particle distance. A similar effect is also known from the de Gennes narrowing [1]. Due to this small separation of time scales for $\hat{\Phi}_{q}(\hat{t})$ at $q_{0}$, the Markovian approximation to 

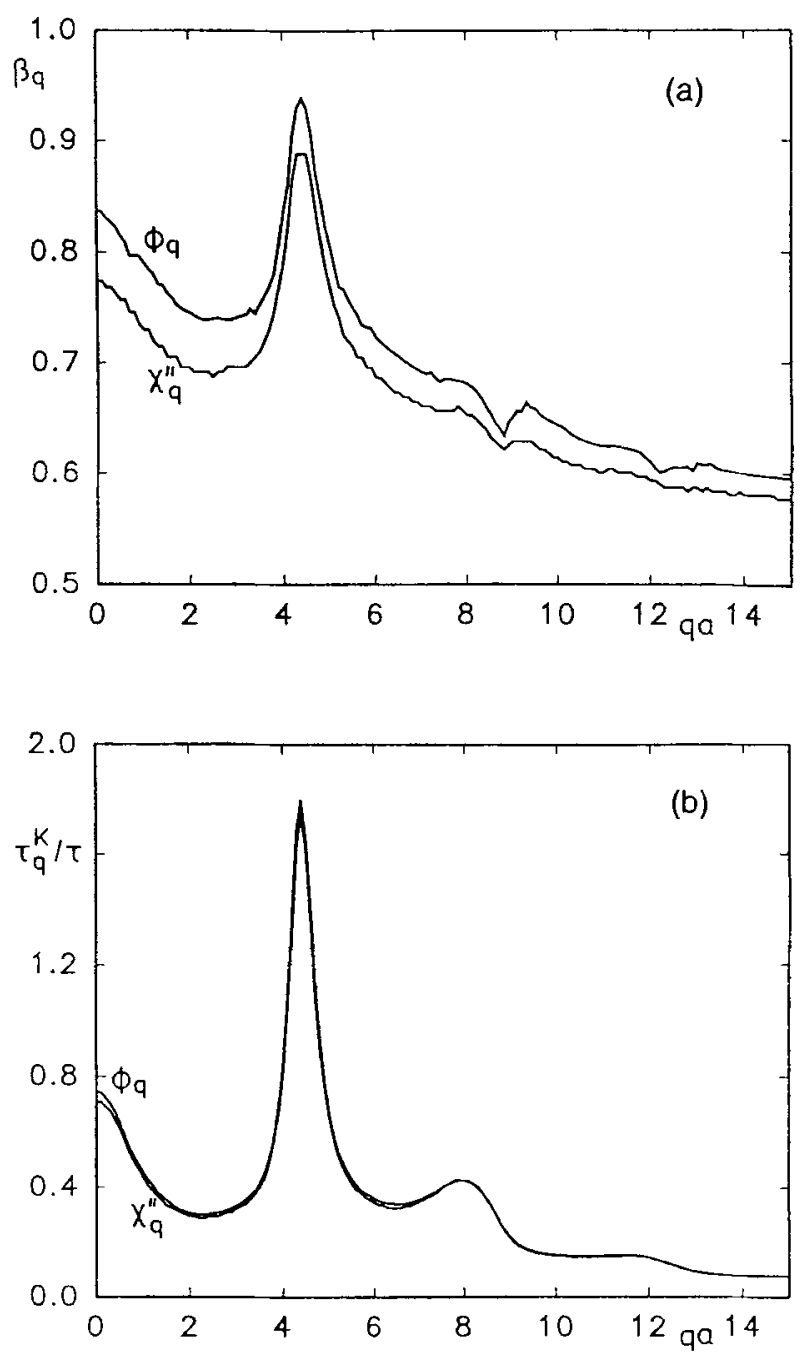

Fig. 9 (a) Kohlrausch exponents $\beta_{q}$ for the coherent density fluctuations versus $q a$ with $a \approx 0.62 d$. The curve labelled $\chi_{q}^{\prime \prime}$ was determined from the logarithmic half width of the peak in the $\alpha$ - susceptibility $\hat{\chi}_{q}^{\prime \prime}(\hat{\omega})$; see [14] for more details.

(b) Kohlrausch relaxation times $\hat{\tau}_{q}$ of the coherent density fluctuations; 
$\hat{m}_{q_{0}}(\hat{\omega})$ works relatively well around $\hat{\omega} \hat{\tau}_{q_{0}} \approx 1$ and the resulting $\alpha$-master function $\hat{\Phi}_{q_{0}}(\hat{t})$ exhibits rather little stretching, $\beta_{q_{0}}=0.89$.

In contrast to the localized $\beta$-process the $\alpha$-process includes transport over large distances. The mean square displacement $W(t)$ defined by [16]:

$$
\left.W(t)=\frac{1}{6}\left\langle\left(\vec{r}_{(} t\right)-\vec{r}(0)\right)^{2}\right\rangle,
$$

can be obtained from the memory function $\tilde{M}_{q=0}^{s}(t)$ by integration. In Fig. 10, $W(t)$ grows linearily in time for long times or large distances. This is expected from an hydrodynamic diffusive description, $W(t) \rightarrow D^{s} t$, for $t / \tau \rightarrow \infty$. Sublinear diffusion results from the tendency of $W(t)$ to arrest at the finite value $W_{c}$, which is given by the localization length discussed in chapter $3, W_{c}=\sqrt{\left\langle\Delta r^{2}\right\rangle} / 2$. The $\beta$-scaling law describes the slowing down of $W(t)$ around $W_{c}[3]$. During the $\alpha$-process the mean square displacement starts to grow, starting from $W_{c}$ like:

$$
W(t)-W_{c}=h_{W}(t / \tau)^{b} \quad \text { for } t_{\sigma} \ll t \ll \tau,
$$

and then smoothly crosses over to the hydrodynamic linear asymptote.

The contents of the $\alpha$-master functions for small and intermediate wave vectors $q$ is tightly connected to the static structure factor. The generally good fit of the Kohlrausch function may also be reasoned. It interpolates in between the fractal high frequency and the regular low frequency wing exhibited by the $\alpha$-master spectra. Yet the observed trend of these fits to improve with increasing $q$ and the trend $\beta_{q} \rightarrow b$ for $q \rightarrow \infty$ sheds more light on the description of the $\alpha$-process offered by the idealized MCT. From probability theory Levy's generalization of the central limit theorem must be mentioned [27]. He considered stochastically independent processes $x_{i}$ whose (identical) distribution function $f(x)$ he characterized by an exponent $\alpha$ with $0<\alpha \leq 2$. Leaving $f(x)$ otherwise totally unspecified $\alpha$ only has to determine the power law tail of the density $f(x)=\mathcal{O}\left(x^{-1-\alpha}\right)$ for $x \rightarrow \infty$. Levy then could prove that the appropriately normalized $\operatorname{sum} X=\frac{1}{a_{N}}\left(x_{1}+\ldots+x_{N}\right)$ in the limit $N \rightarrow \infty$ possesses a probability distribution $F(X)$ which is uniquely determined by $\alpha$. In the case of $\alpha=2$, the well known standard central limit theorem is obtained with $F(X)$ given by the Gaussian distribution function. In this case, the characteristic function $F(t)$ corresponding to $F(X)$ via $F(t)=\int d X e^{i X t} F(X)$ clearly is a Gaussian as well. For $\alpha<2$ however the characteristic function $F(t)$ is given by the Kohlrausch function with exponent $\alpha, F(t)=e^{-t^{\alpha}}$ [27]. In probability theory, therefore, the 


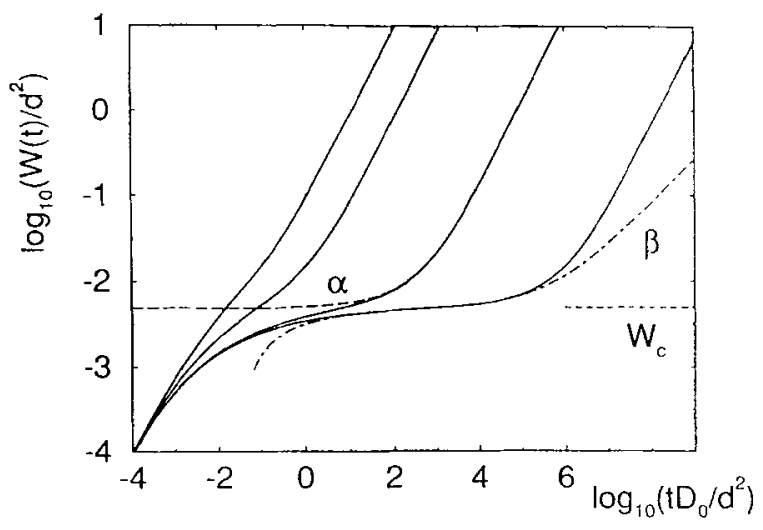

Fig. 10 Mean square displacements for interacting Brownian particles at different packing fractions $\varphi ;(\varphi, \sigma)=(0.437,-.20),(0.494,-.07),\left(0.522,-6.110^{-3}\right)$ and $\left(0.5245,-3.510^{-4}\right)$. The value at localization $W_{c} \approx 510^{-3}$ (dotted line), the result from the $\beta$-scaling law (chain curve, with the critical amplitude $h_{W} \approx 0.01$ ) and the $\alpha$-master curve (broken line) are included. The $\alpha$ - and $\beta$-time scales, $t_{\sigma}$ and $\tau$, are known from (5) and (13) respectively.

Kohlrausch function plays an important role as the generalization of the Gaussian function. It is the only other possible characteristic function which can be obtained by a limiting procedure summing up stochastically independent processes.

In the MCT $\alpha$-equation (20), the correlators $\hat{\Phi}_{q}(\hat{t})$ may be envisaged as characteristic functions corresponding to the probability densities $\hat{\Phi}_{q}^{\prime \prime}(\hat{\omega})>0$. Of course the $\alpha-$ master equations are coupled integral equations. This shows that the assumption of stochastically independent processes underlying the $\alpha$-process fails in general. There are the discussed effects of the static structure factor on the results for $\hat{\Phi}_{q}(\hat{t})$. However, the essential condition for obtaining generalizations of the Gaussian relaxation $e^{-\gamma t^{2}}$, namely the von Schweidler high frequency asymptote, is shared by all $\alpha$-master functions, $\hat{\Phi}_{q}^{\prime \prime}(\hat{\omega}) \propto h_{q}(\hat{\omega} \hat{\tau})^{-1-b}$ for $\hat{\omega} \hat{\tau} \gg 1$ [4]. The connection to Levy's central limit theorem can be established, because, in a limiting case, the Kohlrausch law becomes a solution of the $\alpha$-master equations (20) [28]:

$$
\hat{\Phi}_{q}(\hat{t}) \rightarrow f_{q}^{c} e^{-\left(\bar{t} / \hat{\tau}_{q}\right)^{b}} \text {, with }\left(1 / \hat{\tau}_{q}\right)^{b} \rightarrow q / q^{*} \quad \text { for } \quad q \rightarrow \infty \quad \text { and } \hat{t} \ll 1 \text {. }
$$




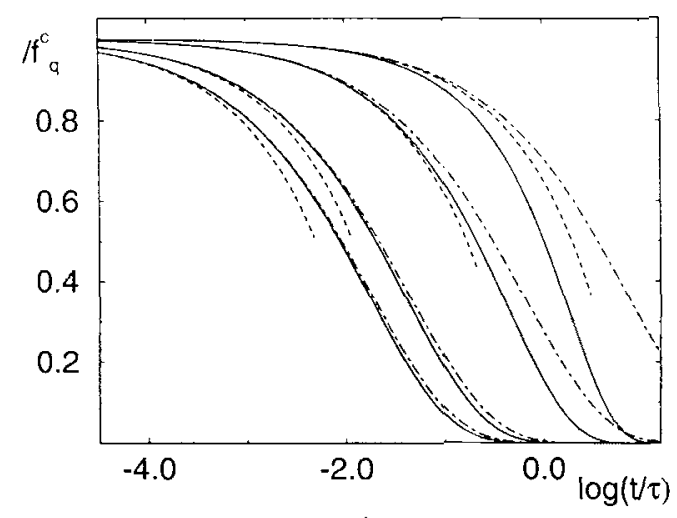

Fig. 11 Comparison of the $\alpha$-correlators $\hat{\Phi}_{q}(\hat{t})$ (solid lines) to the Kohlrausch approximation (23) (chain curves) and the von Schweidler asymptote (short dashes); from right to left the $q$ - values increase ( $q a=4.4,7.8,20,29.9$; with $a \approx 0.62 d$ ) [28].

For large wave vectors, the correlations imprinted by $S_{q}$ onto the $\alpha$-master equations (20) drop out and the general result (23) can be obtained. As required by Levy's central limit theorem, the Kohlrausch exponent equals the von Schweidler exponent in this case. The restriction $\hat{t}$ not too large is not severe in (23) because the divergence of the rate $\left(1 / \hat{\tau}_{q}\right)^{b} \propto q$ for $q \rightarrow \infty$ makes $\hat{\Phi}_{q}(\hat{t})$ relax to zero within the specified time window. Fig. 11 compares the result (23) using the independently determined rate $\left(1 / \hat{\tau}_{q}\right)^{b}=h_{q} / f_{q}^{c}$ with the numerical results for $\hat{\Phi}_{q}(\hat{t})$ for some wave vectors. The expected trend of (23) to improve for larger $q$ is apparent. For hard spheres, the slow decay, for $q \rightarrow \infty$, of the oscillations in $S_{q}$, leads to rather strong deviations. In a mixture of soft spheres the corrections to (23) are smaller [28].

This result suggests the following interpretation of the $\alpha$-process in the idealized MCT. In a first approximation it can be envisaged as resulting from a superposition of stochastically independent processes all sharing the same von Schweidler law (19). Such a neglect of the coupling in the equations (20), however, in general fails to explain the $\hat{\Phi}_{q}(\hat{t})$ for finite wave vectors, where the static structure, as described by $S_{q}$, strongly influences the $\alpha$-relaxation. As discussed for wave vectors $q$ of the order of $q_{0}$, the relaxation times $\hat{\tau}_{q}$ and stretching exponents $\beta_{q}$ are directly correlated to $S_{q}$ 


\section{Acknowledgements}

The fruitful and pleasant collaboration with A. Latz is gratefully acknowledged. The author thanks W. Götze, A. Latz and W. van Megen for helpful discussions and a critical reading of the manuscript. The work was supported by the Deutsche Forschungsgemeinschaft under contract Go 154/8-1.

\section{References}

[1] J.P. Hansen and I.R. McDonald, Theory of Simple Liquids, 2nd edn. (Academic Press, London, 1986).

[2] P.N. Pusey, Liquids, Freezing and the Glass Transition, J.P. Hansen et al. eds. (North-Holland Publ. Comp., Amsterdam, 1991) p. 763.

[3] W. Götze, Liquids, Freezing and the Glass Transition, J.P. Hansen et al. eds. (North-Holland Publ. Comp., Amsterdam, 1991) p. 287.

[4] W. Götze and L. Sjögren, Rep. Prog. Phys. 55, 241 (1992).

[5] R. Schilling, in Disorder Effects on Relaxational Processes, edited by R. Richert and A. Blumen (Springer, Heidelberg, in press).

[6] J.P. Boon and S. Yip, Molecular Hydrodynamics, (McGraw-Hill, New York, 1980).

[7] M. Fuchs, W. Götze, S. Hildebrand, and A. Latz, J. Phys.: Condensed Matter 4, 7709 (1992)

[8] W. van Megen and S.M. Underwood, Phys.Rev.Lett. 70, 2766 (1993).

[9] E. Leutheusser, Phys.Rev. A29, 2765 (1984).

[10] U. Bengtzelius , W. Götze and A. Sjölander, J.Phys. C 17, 5915 ( 1984).

[11] E. Leutheuser, J.Phys. C: Solid State Phys. 15 2801, 2827 (1982).

[12] W. Götze, Philos.Mag. B 43, 219 (1981); in Recent Developments in Condensed Matter Physics Vol. 1, edited by J.T. Devreese (Plenum, New York, 1981) p. 133.

[13] J.L. Barrat, W. Götze and A. Latz, J.Phys.: Condensed Matter 1, 7163 (1989). 
[14] M. Fuchs, I. Hofacker and A. Latz, Phys.Rev. A 45, 898 (1992).

[15] W. Götze and L. Sjögren, Phys.Rev. A 43, 5442 (1991).

[16] W. Hess and R. Klein, Adv.Physics 32, 173 (1983).

[17] B. Cichocki and W. Hess, Physica 141A, 475 (1987).

[18] J.A. Marquese and J.M. Deutch, J.Chem.Phys. 73, 5396 (1980).

[19] B.J. Ackerson and L. Fleishmann, J.Chem.Phys. 76, 2675 (1982); S. Hanna, W. Hess and R. Klein, Physica 111A, 181 (1982).

[20] W. Götze, Z.Phys. B 60, 195 (1985).

[21] M. Fuchs, W. Götze, S. Hildebrand and A. Latz, Z.Phys. B-Condensed Matter 87, 43 (1992).

[22] W. van Megen and S.M. Underwood, Phys.Rev. E 49, 4206 (1994).

[23] H. Löwen, T. Palberg and R. Simon, Phys.Rev.Lett 70, 1557 (1993).

[24] C.H. Bennet, J.Appl.Phys. 43, 2727 (1972).

[25] W. Götze, Z.Phys B 56, 139 (1984).

[26] W. Götze, in Amorphous and Liquid Materials, edited by E. Lüscher (Martinus Nijhoff, Dordrecht, 1987) p. 34.

[27] W. Feller, An Introduction to Probability Theory and its Applications, vol II, 2nd ed. (Wiley, New York, 1971).

[28] M. Fuchs, J.Non-Cryst.Solids, to be published (1994). 